\title{
Analysis of the calculated and experimental dependencies of the combustion of coal dust on the basis of a new methodological base of theoretical studies of heat exchange processes
}

\author{
Evgeniy V. Toropov, Konstantin V. Osintsev, Sergei V. Aliukov* \\ South Ural State University, 76 Prospekt Lenina, Chelyabinsk 454080, Russia
}

Corresponding Author Email: alysergey@gmail.com

https://doi.org/10.18280/ijht.360411

Received: 24 May 2018

Accepted: 5 December 2018

\section{Keywords:}

fuel combustion, coal dust, burning of coal particles, heat exchange

\begin{abstract}
The methodology of theoretical studies of heat exchange processes in boiler units based on the mathematical model of burning coal dust has been developed. For the first time, the continuous curve of the fractional composition of an ensemble of coal particles is presented. The mathematical model is consistent with the aeromechanical and thermal characteristics of the torch, obtained on the basis of calculated and experimental data. The concept of a torch continuum is introduced as a continuous medium in which the processes of combustion and heat exchange are investigated. The methods of dividing the combustion chamber into zones, the equations of stationary and non-stationary heat conduction, radiation and convective heat exchange are used. These methods have been tested on a number of high-temperature installations, including boilers with natural circulation, for example, E-220 at the CHP plant in Bishkek, Kyrgyz Republic in the Central Asian region. In the course of the experiments, coals with different thermal characteristics were burned. In a special computer program obtained a 3D image of burning coal dust. Analysis of the calculated and experimental data showed a discrepancy between them of $4-5 \%$. Analysis of computer simulation data showed a discrepancy with the calculated data of $3-4 \%$, and with experimental $5-6 \%$. Thus, the dependence of the parameters of the heterogeneous flame on the mathematical description of the fractional composition of coal dust is substantiated and the characteristics of the combustion process, previously not taken into account in the theory of heat transfer, are revealed. The task was solved - theoretically confirmed new methodological approaches to the theory of heat transfer in the furnace of the boiler unit.
\end{abstract}

\section{INTRODUCTION}

Studies of the combustion of solid fuels are conducted by scientists in many countries. The most complete picture of the preparation of coal dust at thermal power plants can be obtained in the article [1]. One of the main obstacles for efficient and high-quality solid fuel combustion is its polyfractional composition, which is confirmed by the mathematical models of the authors [2]. Here the most important condition for complete combustion is the presence of a sufficient amount of air as an oxidizing agent and the fineness of grinding coal dust, as noted by the researchers [3].

There are various models of combustion [1] and vibration preparation of solid fuel [3]. In addition, one should not forget the fundamentals of the theory of practical combustion and mathematical modeling, which were developed in the twentieth century in the world of D.B. Spalding [4], and in the countries of Central Asia Y.B. and V.V. Pomerantsev [5]. D.B. Spalding [4] presents the fundamentals of chemical reactions of combustion, physical and mathematical combustion, the development of a torch in the furnace space of a boiler, forecasting the behavior of a torch of various configurations and various fuels. V.V. Pomerantsev subsequently [5] in his work developed the basis for the practical use of accumulated knowledge at specific hightemperature facilities, while he uses data from world scientists, including the American professor F.A. Williams [6]. Under the conditions of lack of natural gas in individual states of Central Asia, scientists supported the main ideas of world scientists and in 2000-2010 began to develop in parallel new methodological approaches to solving the problems of burning lignites, one of the main works is [7]. Finally, in this paper [7], the topic of dust sifting was first raised, which was later reflected in the already mentioned paper [3].

Fuel combustion and heat exchange in the sections of a boiler unit (BU) are optimized and harmful emissions are minimized at the input of the BU gas path and in the combustion preparation system [3]. The development of the methodological basis for heat transfer in boilers is impossible without the introduction of new spatial and geometric characteristics of the boiler unit, which is discussed in [8], where a mathematical model of the combustion process is proposed. Scientists encountering in practice the incomplete burning of coal dust revealed empirical thermal and aeromechanical coefficients [7]. Finally, the scientists came to the conclusion about the expediency of dividing the combustion chamber into separate zones $[7,8]$. In turn, the BU arrangement and the fuel type must be adjusted to the system for preparing and supplying combustion components, the design of fuel-burning appliances (FBA), and the design of the intense combustion zone (ICZ). Thus, the system for preparing and supplying the combustion components and the FBA design provides the conditions for stable ignition and effective fuel burn-out in the ICZ, which is related to the flame temperature and heat exchange in the ICZ [7]. 
Studies by authors such as L. Rosendahl support the theory of weak methodological assurance of heat exchange processes in high-temperature installations; this researcher, together with M. Mando, published an article with his own mathematical model [9]. In [9], the simulation of the jet outflow process consisting of a mixture of air and coal dust was considered. The process of fuel preparation is described in some detail, namely, its grinding, mixing and feeding into the furnace through low-emission burners. In this part, the physical modeling is well analyzed, which is also accompanied by the division of the combustion chamber of the boiler into zones, as in [7]. In addition, in the same article, the authors consider mathematical modeling, on the basis of which the computer modeling process is built.

In the article [8], T. Asotani and his co-authors also consider a model of the behavior of coal particles mixed with air, and the proposed mathematical model correlates well with [9], as well as with some propositions from the authors of $[5,6]$. The mathematical models proposed in the review do not contradict each other, as well as the fundamental laws of physics, and heat transfer in particular.

The disadvantage of all these works is the consideration of reagents as separate structural parts of the combustion medium, which leads to particular methods [10]. In the theory of heat transfer, a new unified methodology is needed based on a mathematical model that includes the design features of the boiler, the torch continuum as an indivisible whole, and the continuous function of the fractional residue.

On the basis of the foregoing, the influence on the process of heat exchange in the furnaces of boiler units of the polyfractional composition of solid fuels should be noted, which was noted in [3]. Analytical solution of the problem of this influence on the combustion process is possible only when developing new mathematical models $[8,9]$. In addition, it is required to develop new methodological approaches to solving this problem, in particular, to divide the boiler into separate zones, and also to consider the combustion medium as a continuously moving torch continuum [7].

In the "Problem Statement" section, the main features of the calculation of an ensemble of coal particles are described and the difficulties of using existing models of sifting coal dust, in particular the Rosin-Rammler model, are noted. The mathematical foundations of the representation of a polyfraction ensemble of coal particles in the form of a monotonically decreasing function are given. There are difficulties in using a monotonously decreasing function under real conditions of sieving coal dust. In the "Development of Fundamental Mathematics" section, the dispersion, the normal distribution function of coal particles and the density of the sieving of particles using the Gauss curve are considered. The solution of similar problems using the Gauss curve is described and a comparison with the works of other authors is given. The section "Adapting the Model to Flame Characteristics" solves the problem of adapting the mathematical model to the actual conditions of sieving coal dust and burning it in a boiler unit using unsteady thermal conductivity and error integrals for the normal distribution function of coal particles. Further, in the section "Dynamics of Particle Combustion", questions of the diffusion-kinetic theory of heat transfer during the burning of coal dust are considered, taking into account the torch continuum as a continuous and inseparable medium. The same section discusses issues of the combustion process related to the concentration of the substance, heat transfer coefficient, the length of the initial part of the torch continuum, temperature and the rate of burning of particles in the torch continuum. Finally, in the "Practical Application" section, the results of experimental studies of the combustion process, the results of computer simulations of the combustion process are given, taking into account new methodological approaches to the study of heat transfer. A comparison is made of the calculated, experimental data and computer simulation data.

\section{PROBLEM STATEMENT}

The widely applied analysis of the particle-size distribution of fuel dust is based on calculating the total residue according to the Rosin-Rammler formula $R_{\delta}=\exp (-$ $\left.b \delta^{n}\right)$, where $\mathrm{b}$ and $\mathrm{n}$ are constant coefficients which characterize the comminution fineness and the homogeneity of the grain-size distribution of fuel dust, respectively. This formula is also used for computer simulation of combustion processes [9]. Application of the total residue characteristic $R_{\delta}$ facilitates reducing all dust fractions to $1.0[4,7]$, taking into account the losses in the analysis, which is consistent with the mass conservation law. This analysis is also consistent with the accepted standard method for sizing disperse solid particles $[10,11]$ and is widely applied.

But, when moving in the flame continuum during heating, volatilization, and burning of volatiles and coke, the fuel dust flows in as a mixture according to its particle size, smallsized particles of $\delta_{\text {min }}$ being the first and large-sized particles of $\delta_{\max }$ being the last. Therefore, to analyze the thermal processes in the flame continuum, it is necessary to relate the analysis of the total residue to the local particle size value in the ensemble of fuel particles $F\left(\delta_{i}\right)$.

The generally applied method is based on the analysis of particle-size distribution in the range of $\delta$-value between $\delta_{1}$ and $\delta_{2} F\left(\delta_{1} / \delta_{2}\right)$, which corresponds to the discrete process of sizing the fuel dust. To obtain a continuous dependence of $F\left(\delta_{i}\right)$, it is necessary to choose a representative point for the interval $\left(\delta_{1} ; \delta_{2}\right)$. The method for particle size analysis recommended in [10], where $F(\delta+\Delta \delta)$ is divided by the variation interval in the particle-size distribution from $\delta$ to $\Delta \delta$, that is, by $\Delta \delta$, is not adequate to the research task, since, according to the definition, $F[\delta /(\delta+\Delta \delta)]=R(\delta)-R(\delta+\Delta \delta)$, and [11].

$$
\frac{F[\delta /(\delta+\Delta \delta)]}{\Delta \delta}=\frac{R(\delta)-R(\delta+\Delta \delta)}{\Delta \delta}=\frac{\exp \left(-b \delta^{n}\right)-\exp \left(-b(\delta+\Delta \delta)^{n}\right)}{\Delta \delta} .
$$

For the value of the total residue $R(\delta)$ that monotonically decreases with the increasing $\delta$, formula (1) yields a relative decrease in the total residue in the interval $[\delta, \delta+\Delta \delta]$, which is conventionally determined in fractions of the total fuel mass. This means that formula (1) yields the variation in the total residue with the change $\Delta \delta$. This is the rate of change of the total residue $R(\delta)$ in the interval of variation $\delta$ with a change of this interval, which is almost unrelated to the change in the residue of coal dust particles in this interval.

According to well-known mathematical dependencies, for example [12], the confidence values of the argument of a monotonically decreasing function can be determined in various ways. At the suggestion of the authors, the function must be presented as continuous, which the researchers did not use in the sieve analysis either in theory or in practice. 
The continuous function allows you to carry out operations such as differentiation and integration with it.

It is rational to use the arithmetic mean value of two intervals $F\left(\delta_{1} / \delta_{2}\right)$ and $F\left(\delta_{2} / \delta_{3}\right)$ to determine the representative value of the residue of coal dust particles in the interval of size change $\delta$. There is a relation $y_{s}{ }^{m} \leq y_{g}{ }^{m} \leq y_{a r}{ }^{m}$ between the arithmetic mean of any value $y_{a r}{ }^{m}$, the geometric mean value $y_{g}{ }^{m}$, and the harmonic mean value $y_{s}{ }^{m}$ [12]. Therefore [13].

$$
F_{i}=0,5\left[F\left(\delta_{1} / \delta_{2}\right)+F\left(\delta_{2} / \delta_{3}\right)\right]=0,5\left[\exp \left(-b \delta_{1}^{n}\right)-\exp \left(-b \delta_{3}^{n}\right)\right]
$$

The representative points $F_{i}$ thus obtained make it possible to plot a continuous curve of the particle-size distribution of the fuel particle ensemble, which allows application of differential and integral calculus to mathematically describe the behavior of particle ensemble in a furnace. The reliability of this approximation, that is, the adequacy of replacing the step function $F[\delta /(\delta+\Delta \delta)]$ with the continuous curve $F_{i}$, increases due to the increase in the number of measurements taken with screens for sizing fuel dust. It is also noteworthy that a sufficiently large number of particles correspond to a single point in the particle-size distribution; since $1 \%$ of the weighed sample mass, equal to $25 \mathrm{~g}$ and $200 \mu \mathrm{m}$, corresponds to 330 particles of this size.

Figure 1 shows a screen for screening coal dust. Figure 2 shows a rotating coal (biofuel) vibrating screen.

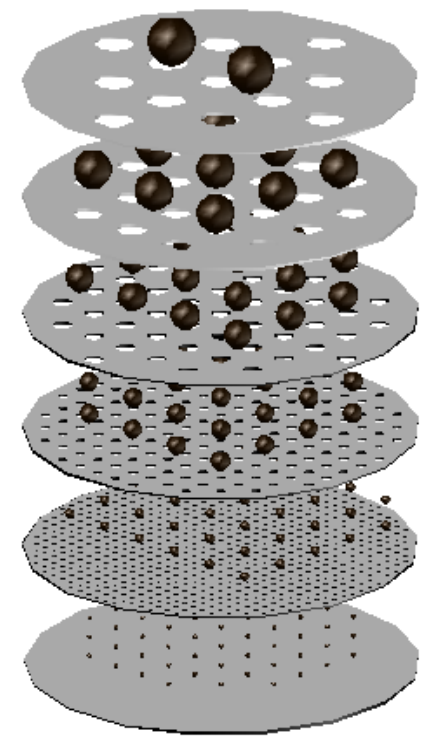

Figure 1. Screen for screening coal dust

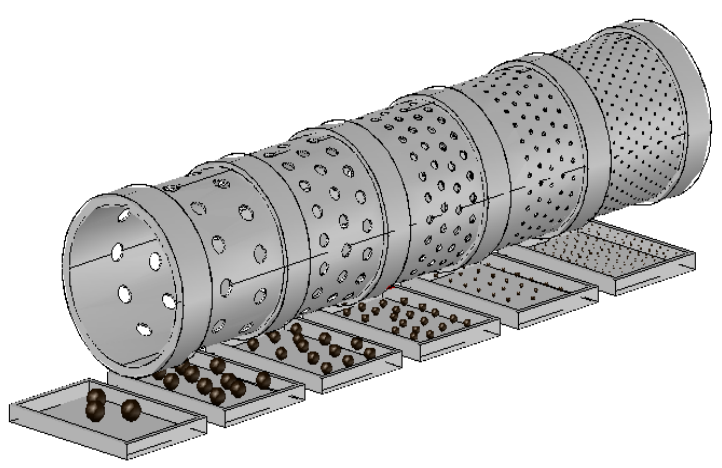

Figure 2. Rotating coal (biofuel) vibrating screen

\section{DEVELOPMENT MATHEMATICS

The qualitative analysis in the framework of the special probability distribution theory [14] shows that the particlesize distribution of solid fuel dust can be described by the equation of hypergeometric distribution, which leads to a binomial distribution if the number of measurements is increased. As shown above, when transformed to the continuous probability distribution, it corresponds to the Gauss curve with the density of normal distribution of probability to deviate from the mean value [15]

$\phi(u)=\exp \left(-0,5 u^{2}\right) / \sqrt{2 \pi}$,

where the standard normal deviation $u=(\delta-\xi) /(\xi \cdot \sigma)$ is introduced as the argument; $\xi$ corresponds to the particle size $\delta$ marking the maximum on the curve $F_{i}=f(\delta) ; \sigma^{2}$ is the dispersion, $\sigma$ is the deviation standard. Normalization in this case means placing the ensemble of fuel particles according to their number near the maximum $\xi$ and reducing all values to the dimensionless form; standardization is reducing the ensemble characteristics to the form of dependence on the deviation standard $\sigma$. The error function $\varphi(u)$, which in this case should be referred to as the deviation function, is tabulated and given in the reference books [14-15]. Figures 3 and 4 show the deviation function for the conditions of the research problem with help of different graphs.

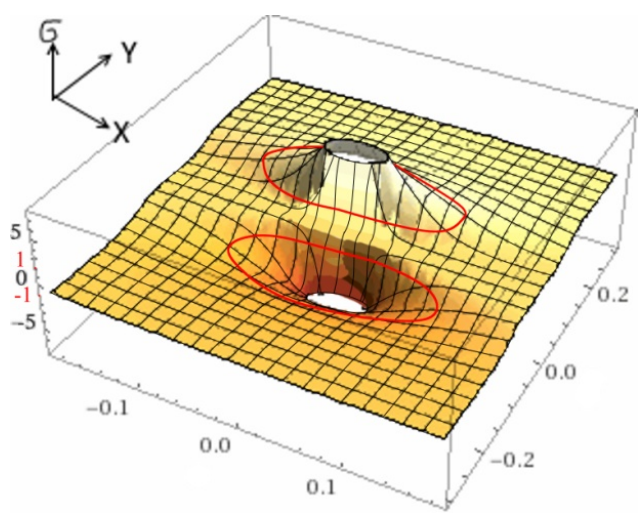

Figure 3. The Gaussian distribution density of the normalized random variable $y=\varphi(u)$

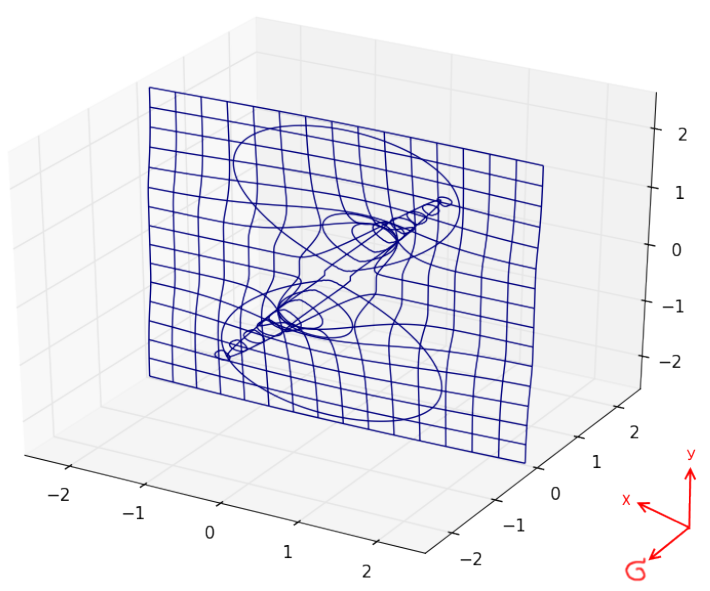

Figure 4. Hodograph of the Gaussian distribution density of the normalized random variable

$$
y=\varphi(u)
$$


It is noteworthy that the standard deviation as a generalizing argument uniquely determines function (3) and the values $u=1$ and $u=-1$ correspond to inflection points on the curve plotted according to the dependence $(3) . \sigma^{2}=(\delta-$ $\xi)^{2} /(\xi \cdot u)^{2}$. Furthermore, the curve $\varphi(u)$ plotted in accordance with the particle-size distribution makes it possible to easily determine the dispersion $\sigma^{2}=(\delta-\xi)^{2} /(\xi \cdot u)^{2}$.

We calculated the correlation coefficient $r_{y 1, y 2}$ [14-15] that characterizes the tightness of the close linear connection between $y_{1}$ and $y_{2}$ to determine how reliable the equation of the Gaussian curve (3), plotted according to the proposed technique $y_{\text {calc }} \equiv y_{2}$, was for the description of the practical particle-size distribution of fuel dust $y_{p r} \equiv y_{l}$. The indices $y_{c a l c}$, $y_{p r}$ mean "calculated" and "practical". Thus, it is rational to subject the dependence (3) to the logarithmic linearization [15].

$$
y_{2}=\ln \phi(u)=-\left(0,92+0,5 u^{2}\right) \text {. }
$$

In papers [14-15], they try to solve similar mathematical problems, as in the sieving of particles. Having determined the correlation coefficient [16-17] $-1,0 \leq r_{1,2} \leq 1,0$ between the practical results of fuel dust sizing and the Gaussian curve in a conventional way [18], we quantified the reliability of description (3). In [19], a solution was proposed for solving similar problems using computer simulation, and in [20-21], the authors approach the exact determination of the desired quantities using specific methods, but they also get quite realistic and practically applicable results. This technique can also be applied at the $F\left(\delta_{i}\right)$ determination stage.

The ensemble of fuel particles, described using the mathematical apparatus proposed in formulas (1) - (3), moves with the air flow at the velocity $w_{l}$ that varies along the flame length $l$ due to the flame expansion and the change in its temperature and dimensions through fuel heating and burn-out. The flame expansion can be registered by the drop in the average kinetic energy $E_{l} / E_{0}=0,56 d_{0} / 0,15 l$ of the circular jet with the exit diameter of the fuel-burning device $d_{0}$ [22-23]. Then the local velocity of the flame continuum without taking into account the change in its temperature is the following $w l^{2}=3,73 w_{0}^{2} d_{0} / l$, whence it follows that $w_{l}=$ $1,93 w_{0}\left(l / d_{0}\right)^{-0,5}$. Similar dependencies are proposed for flat and swirling flames.

The temperature factor in the velocity variation can be taken into account as a first approximation basing on the following relations. Since $E_{l} / E_{0}=\rho w^{2} / \rho_{0} w_{0}^{2}$, the temperature dependence can be determined as $E_{l, T} / E_{0}=\left(\rho_{0} w_{0} w_{T}\right) /\left(\rho_{0} w_{0} w_{0}\right)$ $=w_{T} / w_{0}$ when the mass $\rho w$ is released in the flux density $\rho w^{2}$, which is constant according to the continuity equation $\rho w=$ $\rho_{0} w_{0}=$ const , and hence $[5,17]$

$$
w_{l, T}=1,93 w_{0}\left(T / T_{0}\right)\left(l / d_{0}\right)^{-0,5} .
$$

Thus, within the scope of the stated problem, the velocity of the flame continuum increases in direct proportion to the increase in its absolute temperature and decreases in inverse proportion to the square root of the relative length in calibers $l / d_{0}$. A more accurate consideration of the temperature factor is possible when calculating the heat of advective mass flows produced by the ejection effect of the flame and the heat released during fuel combustion.

\section{ADAPTING THE MODEL TO FLAME CHARACTERISTICS}

When adapting the relations of the multiple-size fuel flux to the aeromechanics of the flame continuum, it is necessary to define two asymptotic conditions that are associated with the initial and final flame sections [4]. The formula $u_{\text {lim }}=$ $\left(\delta_{\text {lim }}-\xi\right) /(\xi \cdot \sigma)$ is used to determine limiting minimum value of coordinate $u_{\text {lim }}$, corresponding to the size of the fuel dust particle $\delta_{\text {lim }}$, which does not yet participate in heating and ignition, and is not involved in calculation. If we place the flame cross-section $l_{f}=0$ at the point where the fuel flux starts to mix with the air and assume that $\delta_{\text {lim }}=0$ in this cross-section, then $u_{\text {lim }}=-\xi /(\xi \cdot \sigma)$, that is, the location of the initial cross-section of the flame continuum depends on the characteristics of particles of the fuel dust ensemble.

The fuel particles of small thermal mass (the number $B i \leq$ 0.1 ) are heated practically without temperature drop in the particle cross-section, therefore the dimensionless temperature $\Theta$ of a particle with the diameter $\delta_{i}$ can be described by the dependence [6]

$$
\Theta=\exp (-3 B i F o)
$$

thermal conductivity, density, and mass heat capacity of the particle material in regulated units. The numerical analysis shows that the $B i$ number can exceed 0.1 when large fuel particles with a high heat transfer rate are heated. The effect produced by the thermal mass of particles on the temperature field (6) can be taken into account by introducing the thermal mass coefficient [6].

The thermal mass coefficient $m$ of the fuel particle is determined by the relation of the coefficient of thermal conductivity on the fuel particle surface $\alpha$ to the coefficient of thermal conductivity $\lambda$, including only a part of the internal thermal resistance and taking into account the particle shape factor $\varsigma[6]$

$$
\begin{aligned}
& m=1+\alpha \delta / 2 \lambda(\varsigma+2)=\alpha[1 / \alpha+0,5 \delta / \lambda(\varsigma+2)]= \\
& =1+0,5 B i /(\varsigma+2) .
\end{aligned}
$$

To a spherical fuel particle approximation $\varsigma=3$ and $m=1$ $+0.1 B i$ with the maximum value of $B i=1.0$, the thermal mass coefficient in this case is $m=1.10$. If we take the thermal conductivity coefficient of a coal particle equal to $\lambda=$ $0.15 \mathrm{~W} / \mathrm{m} \cdot \mathrm{K}$, we can obtain $B i_{\min }=0.03, m=1.003$ for a particle with a size of $50 \mu \mathrm{m}=0.05 \times 10^{-3} \mathrm{~m}$ and a heattransfer coefficient per particle surface of $\alpha=100 \mathrm{~W} / \mathrm{m}^{2} \cdot \mathrm{K}$, which refers to the field of thermally thin bodies. Under the same conditions, a particle of maximum size $\delta=1000 \mu \mathrm{m}=$ $1 \cdot 10^{-3} \mathrm{~m}$ will correspond to the $B i_{\max }=0.67$, while the deviation from the calculation of the particle temperature field according to the laws of thermally thin bodies will reach $6.7 \%$ with mass ratio $m=1.067$. The time of heating to the specified temperature of intensive volatilization should be calculated according to the formula obtained by taking the logarithm of dependence (7) $[4,6]$

$$
\tau_{\text {int }}=[\delta \rho c m /(2 \alpha \varsigma)] \ln \left(\theta_{0} / \theta_{\text {int }}\right),
$$

where $\theta_{0}=t_{g}-t_{0}$ is the difference in the temperatures between the flue gases $t_{g}$ and the initial temperature of the 
particle $t_{0} ; \theta_{\text {int }}=t_{g}-t_{\text {int }}$ is the difference in the temperature between the flue gases and the temperature of intensive volatilization. As the first approximation, $t_{\text {int }}$ can be considered the ignition temperature of the combustible mixture. When $t_{\text {int }}$ increases, the temperature difference $\theta_{\text {int }}$ decreases and the required time $\tau_{\text {int }}$ increases; according to the results of [24], the drying time of the fuel particle can be ignored.

The gradual start of the fuel particles into the heat exchange processes in the flame and the additive dependence of the total heat content are defined by the integral of the distribution function of the deviation probability (3), and it is rational to set the integration limits from minus $\infty$ to the maximum fuel particle size $\delta_{\max }$ corresponding to the upper limit of integration $U[17,20]$

$$
\begin{aligned}
& \Phi(\delta)=\frac{1}{\sigma \sqrt{2 \pi}} \int_{-\infty}^{\delta_{\max }} \exp \left\langle-0,5[(\delta-\xi) /(\sigma \cdot \xi)]^{2}\right\rangle d \delta= \\
& =\Phi_{u}(U) .
\end{aligned}
$$

Moreover, the normal distribution function $\Phi_{i}(U)$ is associated with the probability integral $\Phi(U)$ and the error function $\operatorname{erf}\left(U / 2^{0,5}\right)$ by the dependence $[17,20]$

$$
\Phi_{i}(U)-1 / 2=0,5 \operatorname{erf}(U / \sqrt{2})=\Phi(U) .
$$

Thus, the normal distribution function $\Phi_{i}(U)$ can be defined by the probability integral $\Phi(U)$ or by the error function $\operatorname{erf}(U / \sqrt{2})$, both dependences are tabulated [14, 17]. But the practical adaptation of these dependences has a number of features that are related to the reliability of the dependences used. These features can be determined by the numerical analysis of the dependences (8) - (9) when changing the variability intervals of the arguments and their functions. Figure 5 shows the distribution function $\Phi_{i}(U)$.

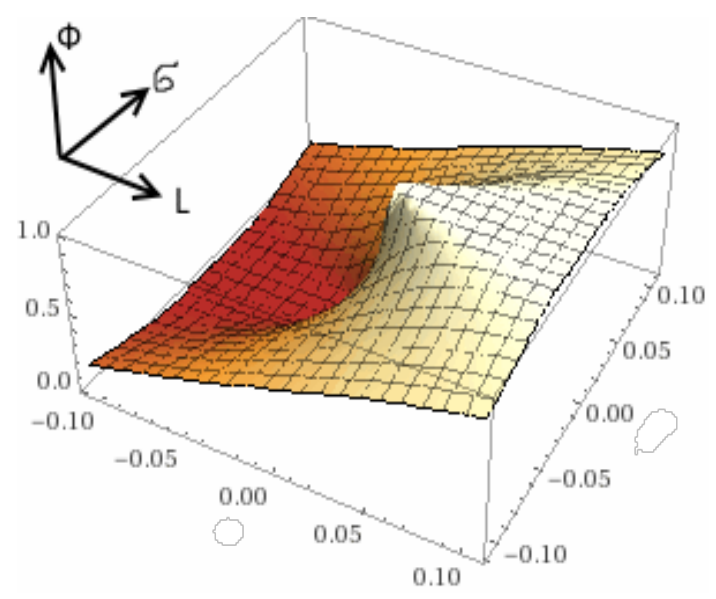

Figure 5. Normal distribution function $\Phi$ of the random variable

$$
x=u=(\delta-\xi) /(\xi \cdot \sigma)
$$

The numerical analysis shows that when the probability integral (9) is used for the range of positive and negative values $u=(\delta-\xi) /(\xi \cdot \sigma)$, the difference between $\Phi(U)$ and $\Phi(\delta)$ does not exceed $0.10 \%$ in the whole variation interval $u$. When calculating $\Phi_{u}(U)$ according to the dependence (10), the difference may exceed $10 \%$ in the range of negative values $u$, which is related to the lower limit of integration when obtaining the error function [17-18],

$\operatorname{erf}(U / \sqrt{2})=2 / \sqrt{\pi} \int_{0}^{\pi} \exp \left(-[U / \sqrt{2}]^{2}\right) d u$

The lower integration limit is determined by the particlesize distribution of the fuel particles in the range of small values $\delta$ and is important for calculating the ignition processes of the air-fuel mixture, and the upper limit is related to the calculation of fuel underburning for the specified flame length, or to the determination of the required flame length for the specified fuel underburning, which is also associated with the particle-size distribution of fuel in the range of large values $\delta$. Similar solutions are presented in $[12,21]$.

The resulting integral function is related to the known probability integral [14,21], which is given in tables in reference books; it makes it possible to calculate not only the total residue of the known particle-size distribution, but also to define a number of properties of the multiple-size fuel flame. Thus, the reduced dependence (8) makes it possible to determine the start of a certain temperature regime depending on the particle size and the conditions of its motion with the multiple-size flame. In order to determine the burnout of fuel particles of different sizes, the adopted model should be complemented with the conditions under which the burning particle interacts with the flux of the furnace and flame medium.

If we assume that the interaction pattern of a burning fuel particle with the furnace atmosphere [23-24] has a surface model, which corresponds to a plane diffusion layer between a spherical particle and the furnace atmosphere, the burnout rate of the particle can be determined at $G_{c}=$ const according to the equation $d \delta / d \tau=-2 M_{c} G_{c} / \rho$, where $M_{c}=12 \mathrm{~kg} / \mathrm{kmol}$ is the molar mass of carbon, $\rho \approx 800 \mathrm{~kg} / \mathrm{m}^{3}$ is the density of coke. The adopted pattern of surface one-dimensional burning in the primary reaction with $\mathrm{CO}_{2}$ generation has the first order in oxygen; combustion is considered quasistationary and self-similar in time. In this case, the total burnout time of a particle with the initial size $\delta_{0}$ is the following [4,23]:

$$
\tau=-\frac{1}{2 M_{c}} \int_{\delta_{0}}^{\delta}\left(\rho / G_{c}\right) d \delta=0,0417 \int_{\delta}^{\delta_{0}}\left(\rho / G_{c}\right) d \delta
$$

If we substitute the integral expression of time $\tau$ in (7) for the process associated with heating and burning of fuel in the flame, we can obtain the distribution function of heat release in the flame along its length. But this pattern requires refinement both in terms of assuming spherical surfaces to be flat, and in terms of the dependence of $G_{c}$ on $\delta$.

To take into account the polydispersity of the medium in the flame continuum, we need to apply the dependence for the single-particle burnout function associated with the local value of the kinetic and diffusive characteristics of the process. As a first approximation, we can neglect the effect of $\mathrm{CO}$ burning within the boundary layer, the reducing reactions, and the internal response [23-24]. Then, a onedimensional model of the combustion process of the firstorder oxygen reaction, with oxygen being insufficient, has the dependence for the burnable carbon flux $G_{c}: G_{c}=\omega C_{o x}$, 
where $\omega$ is the kineticity parameter of the fuel burnout process, determined in accordance with Kirchhoff's second electrical rule for a parallel circuit of two conduction bands. The oxidation function is determined by the amount of oxygen in the flame gas $0.21 P_{g} / R T$ and its consumption for the combustion of volatile substances and carbon in stoichiometric ratios.

\section{DYNAMICS PARTICLE COMBUSTION}

The dynamics of multiple-size fuel combustion is associated with the distribution of temperature and concentration of components along the length of the flame continuum; the application of electrothermal analogy and Kirchhoff's electrical rules for nodes and circuits allows us to relate a number of factors. Marking with nodes the points on the burning particle surface without oxidizer $C_{o x}=0$ and those on the outer surface of the diffusive boundary layer, where the concentration of the oxidizer is equal to that of the flame continuum $C_{o x} f$, we obtain the difference in oxidizer transport potentials $\Delta C_{o x}=C_{o x}{ }^{f}-0=C_{o x}{ }^{f}$. Arranging the distributed diffusive resistance $1 / \alpha_{D}$ parallel to the lumped kinetic resistance $1 / k_{1}$, we obtain the total diffusive-kinetic resistance to the combustion process $R_{\Sigma}=1 / \alpha_{D}+1 / k_{1}=1 / \omega$, where the diffusive-kinetic conductivity $\omega=1 / R_{\Sigma}=\alpha_{D} k_{l} /\left(\alpha_{D}\right.$ $\left.+k_{l}\right)[10]$.

The factor $\omega$ can also be referred to as a kinetic index of the fuel burnout process $\omega_{k}$ for $\alpha_{D} » k_{l}$, when the diffusion processes do not limit the burning rate, $\omega_{k}=1.0$; for $\alpha_{D}$ " $k_{l}$, when the diffusion processes become limiting, $\omega_{k} \rightarrow 0$. For $\alpha_{D}=k_{1} \omega_{k}=k_{1} / 2$, which corresponds to the intermediate regime; thus, applying the kinetic index of the combustion process $\omega_{k}$ we can formally designate the boundaries of the kinetic and diffusion regimes including the intermediate region, which corresponds to the trans-regime representations in the dynamics of the solid-fuel burnout process.

The local concentration of oxygen in the flame continuum $C_{o x}{ }^{f}, \mathrm{kmol} / \mathrm{m}^{3}$, is determined by the initial volumetric concentration of $C_{o x . i}$, associated with the combustion method and the burner with air blast $C_{o x . i}=0.21$, and with oxygen consumption for the combustion of volatiles and coke according to the oxygen balance $[4,10]$

$$
C_{o x}^{f}=\frac{C_{o x . i} P_{T} V_{\kappa}^{0}}{R T V_{g}}\left[(\alpha-1) \frac{V^{0}}{V_{k}^{0}}+K_{H}^{p}\right]
$$

(13) takes into account that the stoichiometric air consumption $V^{0}, \mathrm{~m}^{3} / \mathrm{kg}$, is equal to the air consumption for the combustion of coke and volatiles $V^{0}=V_{k}^{0} K^{c}+V_{v}^{0} V_{v}^{c}$. The oxygen content according to (13) ensures the local burnout of the fuel particle with the initial size $\delta_{i}$ with respect to this dependence without taking into account the variability of the variability of the kinetic factor $[10,23]$

$$
\frac{d \delta_{i}}{d \tau}=-\frac{2 M}{\rho_{\kappa}} \omega_{\kappa} C_{o \kappa}^{f} .
$$

The diffusion coefficient of mass delivery $\alpha_{D}$ is generally determined by the dependence of $N u_{D}$ on numbers $R e$ and $P r$, where the former includes the relative velocity of a particle in the flux. When the flame flux develops, the relative velocity and the Re number reduce to zero, with $N u_{D} \approx 2$. Thus, the kinetic index of the process of fuel particle burning under these conditions is defined by the formula $[5,10]$

$$
\begin{aligned}
& \omega_{\kappa}=k_{1} N u_{D} D / \delta_{i} /\left(k_{1}+N u_{D} D / \delta_{i}\right)= \\
& =2 k_{1} D / \delta_{i} /\left(k_{1}+2 D / \delta_{i}\right) .
\end{aligned}
$$

Taking into account (13) - (15), the combustion velocity of a fuel particle with size $\delta_{i}$ is determined by the formula $[5,10]$

$$
\begin{aligned}
& \frac{d \delta_{i}}{d \tau}=-\frac{2 M}{\rho_{\kappa}} \frac{2 k_{1} D / \delta_{i}}{\left(k_{1}+2 D / \delta_{i}\right)} \frac{C_{\text {ок.и }} P_{T} V_{\kappa}^{0}}{R T V_{g}}\left[(\alpha-1) \frac{V^{0}}{V_{\kappa}^{0}}+K_{u}^{p}\right]= \\
& =-\omega_{\kappa} \frac{B}{T} .
\end{aligned}
$$

Depending on (16), parameter B includes all the constants of the specified task, the proportion of unburned coke $K_{u}^{r}$ is assumed to be the same for the particle of any size, the temperature of the flame continuum $T$ can be related to the abscissa $X$ and the flame length, thus the effect of the particle-size distribution consists in the factor $\omega_{k}$. This allows separation of the differentials and integration of the dependence (16) for obtaining the burnout function of fuel particles of size $\delta_{i}[5,10]$.

$\tau_{v y g}=-\frac{T}{B} \int_{\delta_{i 0}}^{0} \frac{2 D+k_{1} \delta_{i}}{2 k_{1} D} d \delta_{i}=\frac{\mathrm{T}}{\mathrm{B}}\left(\frac{\delta_{i 0}}{k_{1}}+\frac{\delta_{i 0}^{2}}{4 D}\right)$.

The change of sign in (17) is connected with the change in the limits of integration from 0 to $\delta_{i 0}$, which corresponds to the dynamics of fuel particle burnout. The temperature of the flame continuum, which affects the time of fuel particle burnout, is determined here by the approximation from $T_{\text {int }}=$ $t_{\text {int }}+273$ to $T_{\text {con }}$, corresponding to the final flame crosssection $L_{f}=1.0[6,10]$.

$T=T_{i n t}+L_{f}\left(T_{c o n}-T_{i n t}\right)$

The longitudinal dimensions of the flame continuum can be determined using different thermal physic techniques of diagnosis in the field of aeromechanics, temperature and radiation, concentration and analysis, as well as using techniques of mathematical statistics. The method for diagnosis should be selected in accordance with the main research task. In this paper, determination of flame characteristics is based on the dispersion analysis of fuel dust, so it is rational to determine the initial flame section $l_{0}$ by the ignition time of the smallest fuel dust particles according to (8). In this case, $l_{0}$ will differ from the distance to the burner port by the amount that is required to heat and ignite the smallest dust particles.

The main task under the stated conditions is to determine how much the initial velocity at the nozzle outlet $F_{0}=\pi r_{0}^{2}$ is influenced by three main factors: jet expansion due to the involvement of the ICZ medium into the flux, change in velocity due to the change in particle-size distribution of the flux caused by combustion and temperature change of the flame medium from $T_{0}$ to $T_{f}$. Thus, the change in velocity of the flame medium from $L_{f}=0$ to $L_{f}=1.0$ during its motion and combustion process can be represented by the 
dependence $[4,10]$

$$
\mathrm{w}_{f}=w_{0} k_{F} k_{V} k_{T}
$$

where $k_{F}, k_{V}, k_{T}$ are the influences of change in flame crosssection, change in volumetric flow in the flame, and its temperature change, correspondingly $[4,10]$ :

$$
\begin{aligned}
& k_{F}=\frac{1}{\left(1+0,21 l_{f} / d_{0}\right)^{2}} ; \\
& k_{V}=\frac{1}{\left[1+L_{f}\left(\frac{V_{g}^{\alpha}}{\mathrm{V}_{v}^{\alpha}}-1\right)\right]} ; \\
& k_{T}=T_{f} / T_{0}=1+\Delta T_{g o r} L_{f} / T_{0} .
\end{aligned}
$$

The relation (20) is obtained geometrically on the basis of central angles of the heterogeneous flame divergence equal to $12^{0}$ that are experimentally measured taking into account the advective mass fluxes. Relations (21) - (22) admit the linear approximation of the dependence $\Phi=f(X$, ) associated with fuel burnout and temperature rise in the flame, as Figure 3 shows. The factor $l_{f} / d_{0}$ is the distance from the nozzle in calibers. This factor is applied here, because the flame jet advances up to the plane of fuel ignition initiation according to the aeromechanic laws and without internal heat sources. The factor $\Delta T_{\text {com }}$ takes into account the effect of flame temperature increases due to the combustion reaction of fuel.

After the fuel ignition at $L_{f}=0$, particle-size related burnout of fuel and temperature increase of the flame continuum take place in accordance with the integral curve $\Phi$ $=f(X)$. By taking into account this factor, we make the task iterative, therefore the linear approximation $\Phi=f(X)$ is a possible solution, while dependences (20)-(22) describe the second-order influences. In fact, these approximations result from the expansion of the $V\left(L_{f}\right)$ function, as shown below.

The amount of gas phase in the flame continuum $V_{g}, \mathrm{~m}^{3} / \mathrm{kg}$, changes from $\alpha V^{0}$ at the flame starting point with $L_{f}=0$ before the ignition $(\alpha-1)$ to $V^{0}+V_{g}{ }^{\alpha=1}$ at at the flame end when the combustion is completed, where $L_{f}=1.0$. If the function $V_{g}\left(L_{f}\right)$ is expanded in the Taylor series, the first two addends of the series give $[6,10]$

$$
V_{g}\left(L_{f}\right)=\alpha V^{0}+L_{f} \frac{\partial V_{g}^{\alpha=1}}{\partial L_{f}}
$$

We determine the variables $V^{0}$ and $V_{g}{ }^{\alpha=1}$ that form part of formula (23) by the reference data for a specific type of solid fuel [8] at $0 \leq L_{f} \leq 1.0$. Similarly, we determine the flame continuum enthalpy in its temperature change function, and the temperature changes can be determined in the first approximation by the enthalpy change with $c_{r}=$ const .

The determined flame boundaries make it possible to plot the starting and final points of the flame continuum on the Xaxis, and, accordingly, to find the starting and final points of the flame on the $\Phi$-axis, considering it as the heat source and taking into account the initial flame section. The corrections to the flame continuum velocity (19) are applied sequentially: only the correction $k_{F}$ is applied in the range from $l_{f}=0$ to $l_{f}=$ $l_{i}$, after that all three corrections up to the final flame crosssections are applied.

The length of the initial section preceding the ignition initiation $l_{i}$ depends on the initial velocity of the flame flux $w_{0}$ and the heat transfer rate in terms of convection $\alpha, \mathrm{W} / \mathrm{m}^{2} \mathrm{~K}$, which gives the following if only $k_{F}$ is taken into account [6, $10]$

$$
\begin{aligned}
& l_{\mathrm{i}}^{3}+19,0476 l_{\mathrm{i}}^{2}+90,703 r_{0}^{3} l_{\mathrm{i}}= \\
& =0,22576 \cdot 10^{3} w_{0} r_{0}^{2} / \alpha,
\end{aligned}
$$

or if $l_{i}$ is substituted with $l_{i, f}=l_{i} / d_{0}$ and dimensionless similarity numbers $R e=w_{0} d_{0} / v$ and $N u=\alpha d_{0} / \lambda$ are applied $[6,10]$

$$
\begin{aligned}
& l_{i, f}^{3}+9,5238 l_{i, f}^{2}+5,6689 d_{0} l_{i, f}= \\
& =0,04 \cdot 10^{-6} \mathrm{Re} / d_{0} N u .
\end{aligned}
$$

Equation (24) can be solved either by Cardano's method, or numerically; adaptation calculations show that the length of the initial section is $0.95 \ldots 1.30$ calibers, which is consistent with the experimental data.

The transformations of the abscissa, make it possible to numerically determine the function $\Phi$ on the graph ordinate as an integral function of the increase in the flame continuum enthalpy, which can be related to its adiabatic temperature $[6,10]$

$t_{a \partial}=\Phi\left(L_{f}\right) \frac{Q_{p}^{p}}{c_{p} V_{g}}$.

If the available heat of the fuel flux $Q_{p}{ }^{p}$ and the yield of combustion products per the fuel mass unit $V_{g}$ with the heat capacity of the combustion products $c_{p}$ are substituted in the formula (26), the local adiabatic temperature value of the flame continuum $t_{a d}$ can be determined when the obtained result is multiplied by the normal deviation function $\Phi\left(L_{f}\right)$ for the given coordinate $L_{f}$ [25-27]. The transition from $\Phi(U)$ to $\Phi\left(L_{f}\right)$ is realized by calculating the time of the particle-size related burnout of fuel in accordance with (17), which corresponds to the time determined by the actual velocity of particles in the flame $\tau=l_{f} / w_{f}$ according to (19). The obtained dependence is solved with respect to the relation between $l_{f}$ and $u=\left(\delta_{i}-\xi\right) /(\xi \cdot \sigma)$.

\section{PRACTICAL APPLICATION}

As a result of the analytical calculations, the dependence of the parameters of a heterogeneous flame on the mathematical description of the fractional composition of coal dust was substantiated; The characteristics of the combustion process that were previously not taken into account in the theory of heat transfer were revealed; theoretically confirmed new methodological approaches to the theory of heat transfer in the furnace of the boiler unit [28, 29].

The results of theoretical studies were verified by the authors at existing thermal power plants, in particular when burning coal dust in E-220 boilers. In the laboratory of thermal power engineering of the scientific and technical center under the Ministry of Energy of the Kyrgyz Republic, 
comparative analyzes and experimental studies were conducted on samples of coal dust of various compositions. The movement of coal dust through the sampling tubes of devices, as well as the degree of grinding in roller and hammer mills was taken into account. The results showed good convergence within the engineering error of theoretical and experimental data [7].

The length $\mathrm{Lf}=5.1$ meters and the flame temperature at the length of the flame Lf during the operation of the E-220 boiler was $\mathrm{T}=1786 \mathrm{~K}$. The data are given depending on the steam output of the boiler unit at the nominal boiler load Dnom $=61 \mathrm{~kg}$ of steam per second. In addition, the data are given when burning Kyrgyz coal with a heat of combustion of $27,820 \mathrm{~kJ} / \mathrm{kg}$.

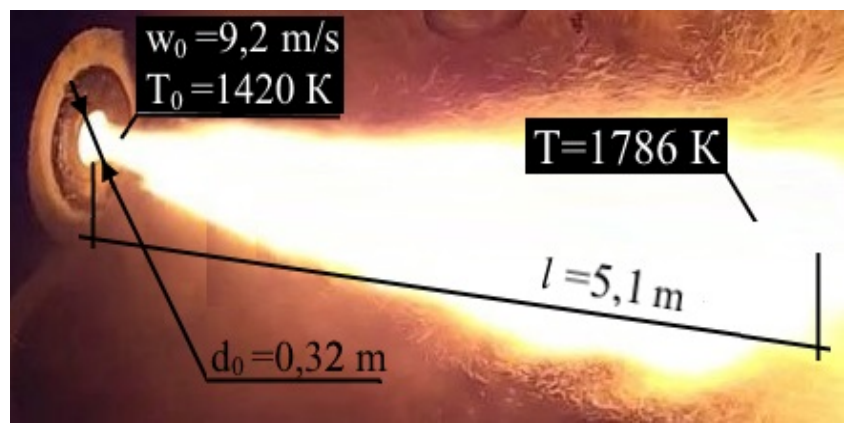

Figure 6. Experimental study of combustion on the boiler E-160

Analysis of the calculated and experimental data showed a discrepancy between them of $4-5 \%$, which could be explained by some error in conducting experiments at high temperatures in boiler installations, for example, re-radiation and high dust plume in the combustion space.

In addition, the possibility of treating the torch as a continual medium was theoretically confirmed; The prospects for identifying the distribution of the adiabatic temperature along the length and height of the torch continuum in the zone of intense combustion of the combustion chamber of the boiler unit are shown.

It should be noted that computer simulation was also carried out in a special program, in which the boundary and initial conditions were taken into account as during the combustion of coal dust in the furnace of the E-160 boiler unit, Fig.7 [7].

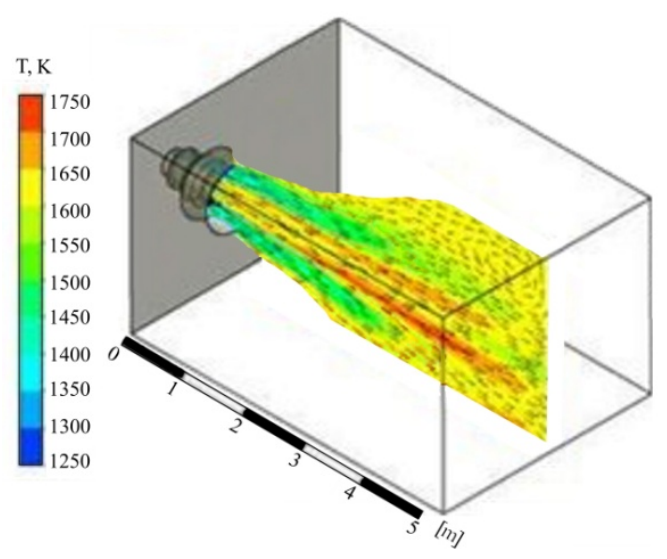

Figure 7. The results of computer simulation
Analysis of computer simulation data showed a discrepancy with the calculated data of $3-4 \%$, and with experimental $5-6 \%$.

\section{CONCLUSIONS}

The possibility of considering the torch as a single continuum, to which the authors refer flue gases in the zone of intense combustion and screen tubes, is theoretically confirmed. The prospects of determining the type of distribution of the adiabatic temperature along the length and height of the flare continuum in the zone of intense combustion of the combustion chamber of the boiler unit are shown.

We applied a new mathematical method to describe the behavior of the polydisperse flux ensemble of fuel particles in a combustion process, which allowed us to determine the function of particle-size related burnout of fuel and the related enthalpy and local adiabatic temperature of the flame continuum.

We determined the length $l_{i}$ of the initial flame section, where the fuel does not ignite yet. It depends on the intensity of heat supply to the initial section $\alpha$, the accepted temperature $t_{\text {int }}$ of intensive volatilization, the velocity of flux $w_{0}$ at the burner exit and its change due to the temperature rise and flame divergence.

The burnout time of $\delta_{i}$-size particles of the multiple-size particle flux depends on the fuel characteristics and the kinetic parameter $\omega_{k}$ of the combustion process, the temperature $T_{f}$ of the flame continuum being taken into account.

\section{ACKNOWLEDGMENT}

The work was supported by the Government of the Russian Federation (Resolution No. 211 of 16 March 2013), contract No. 02.A03.21.0011.

The authors thank the editor and the reviewer for their insightful comments. The authors thank South Ural State University (SUSU) for supporting.

\section{REFERENCES}

[1] Noble A, Luttrell GH. (2015). A review of state-of-theart processing operations in coal preparation. International Journal of Mining Science and Technology 25(4): 511-521. https://doi.org/10.1016/j.ijmst.2015.05.001

[2] Dong L, Zhang Y, Zhao Y, Peng L, Zhou E, Cai L, Zhang B, Duan C. (2016). Effect of active pulsing air flow on gas-vibro fluidized bed for fine coal separation. Advanced Powder Technology 27(5): 2257-2264. https://doi.org/10.1016/j.apt.2016.08.012

[3] Peng L, Wang Z, Ma W, Chen X, Zhao Y, Liu C. (2018). Dynamic influence of screening coals on a vibrating screen. Fuel 216: 484-49. https://doi.org/10.1016/j.fuel.2017.12.041

[4] Spalding DB. (1995). Some fundamentals of combustion. London, Butterworth's, Academic Press, pp. 250 . 
[5] Zeldovich YB. (1992). Collected works. Princeton, Princeton University Press, 1: 500.

[6] Williams FA. (1985). Combustion theory. Second Edition, Benjamin/Cummings, Menlo Park, CA, Westview Press, pp. 704.

[7] Osintsev KV. (2012). Changeover to low-temperature combustion of fuel in the boilers at cogeneration stations in Bishkek. Thermal Engineering 59(8): 598603.

[8] Asotani T, Yamashita T, Tominaga H, Uesugi Y, Itaya Y, Mori S. (2008). Prediction of ignition behavior in a tangentially fired pulverized coal boiler using CFD. Fuel 87(4-5): 482-490. https://doi.org/10.1016/j.fuel.2007.04.018

[9] Mando M, Rosendahl L, Yin C, Sørensen H. (2010). Pulverized straw combustion in a low- $\mathrm{NO}_{\mathrm{x}}$ multifuel burner: Modeling the transition from coal to straw. Fuel 89(10): 3051-3062. https://doi.org/10.1016/j.fuel.2010.05.016

[10] Kyprianidis K.G, Skvaril J. (2016). Developments in Combustion Technology. InTech, Cranfield https://doi.org/10.5772/61418

[11] Yu X, Luo Z, Li H, Gan D. (2018). Effect of vibration on the separation efficiency of oil shale in a compound dry separator. Fuel 214: 242-253.

[12] Leimkulher B, Reich S. (2004). Simulating Hamiltonian Dynamics. Cambridge University Press Cambridge.

[13] Hairer E, Lubich C, Wanner G. (2006). Geometric Numerical Integration. Second ed. Springer Berlin.

[14] Brugnano L, Iavernaro F. (2016). Line Integral Methods for Conservative Problems. CRC Press Boca Raton, FL.

[15] Blanes S, Casas F. (2016). A Concise Introduction to Geometric Numerical Integration. CRC Press Boca Raton, FL.

[16] Sanz-Serna J.M. (2016). Symplectic Runge-Kutta schemes for adjoint equations, automatic differentiation, optimal control, and more. SIAM Review 58(1): 3-33. https://doi.org/10.1137/151002769

[17] Hairer E, Wanner G. (1996). Solving Ordinary Differential Equations II. Stiff and DifferentialAlgebraic Problems. Springer Ser. Comput. Math., 14. Second ed. Springer Berlin.

[18] Brugnano L, Frasca Caccia G, Iavernaro F. (2014). Efficient implementation of Gauss collocation and Hamiltonian boundary value methods. Numerical Algorithms 65(3): 633-650 https://doi.org/10.1007/s11075-014-9825-0

[19] Silva IR, Maboudou-Tchao EM, de Figueiredo WL. (2018). Frequentist-Bayesian Monte Carlo test for mean vectors in high dimension. Journal of Computational and Applied Mathematics 333: 51-64.

[20] Maboudou-Tchao EM, Silva I. (2013). Tests for mean vectors in high dimension. Statistical Analysis and Data Mining 6(6): 578-598. https://doi.org/10.1002/sam.11209

[21] Robbins H. (1951). Asymptotically subminimax solutions of compound decision problems (1951). Proceedings of the Second Berkeley Symposium on Mathematical Statistics and Probability, pp. 131-148.
[22] Robbins H. (1955). An empirical bayes approach to statistics. Proceedings of the Third Berkeley Symposium on Mathematical Statistics and Probability, pp. 157-164.

[23] Spitzner DJ. (2008). An asymptotic viewpoint on highdimensional Bayesian testing. Bayesian Analysis 3(1): 121-160. https://doi.org/10.1214/08-BA305

[24] Inyang HI, Bae S, Pando MA. (2016). Contaminant dust suppression materials: A cost-effectiveness estimation methodology. Measurement: Journal of the International Measurement Confederation 93: 563-571. https://doi.org/10.1016/j.measurement.2016.07.024

[25] Bartholomew A, Fenn R. (2008). Quaternionic invariants of virtual knots and links. Journal of Knot Theory and its Ramifications 17(2): 231-251. https://doi.org/10.1142/S021821650800604X

[26] Carter JS. (1991). Classifying immersed curves. Proceedings of the American Mathematical Society 111(1): 281-287. https://doi.org/10.1090/S0002-99391991-1043406-7

[27] Rodríguez-Nieto JG. (2018). Characterization of signed Gauss paragraphs and skew-symmetric graded matrices. Journal of Knot Theory and its Ramifications 27(1): 1850002 .

[28] Aliukov S, Alyukov A. (2017). Analysis of Methods for Solution of Differential Equations of Motion of Inertial Continuously Variable Transmissions. SAE Technical Paper 2017-01-1105. https://doi.org/10.4271/2017-011105

[29] Camaraza-Medina Y, Khandy NH, Carlson KM, CruzFonticiella OM, García-Morales OF, Reyes- Cabrera D. (2018). Evaluation of condensation heat transfer in aircooled condenser by dominant flow criteria. Mathematical Modelling of Engineering Problems 5(2): 76-82. https://doi.org/10.18280/mmep.050204

\section{NOMENCLATURE}

$\begin{array}{ll}\mathrm{Bi}=\alpha \lambda / \delta ; & \text { Bio dimensionless criterion; } \\ \alpha & \text { heat emission coefficient, } \mathrm{W} /\left(\mathrm{m}^{2} \mathrm{~K}\right) ; \\ \lambda & \text { coefficient of thermal conductivity, } \\ & \mathrm{W} /(\mathrm{m} \mathrm{K}) ; \\ \delta & \text { determining size of the surface, } \mathrm{m} ; \\ \mathrm{Fo}=a \tau / \delta^{2} ; & \text { dimensionless Fourier's criterion; } \\ a & \text { coefficient of thermal diffusivity, } \mathrm{m}^{2} / \mathrm{s} ; \\ \tau & \text { time, } \mathrm{s} ; \\ \mathrm{m} & \text { dimensionless coefficient of thermal } \\ \mathrm{k} & \text { massiveness of the body; } \\ \varsigma & \text { heat transfer coefficient, W/(m K); } \\ N u_{D} & \text { dimensionless particle shape factor; } \\ & \text { dimensionless Nusselt's diffusion } \\ \delta_{i} & \text { criterion; } \\ k_{l} & \text { size of the } i \text {-th particle, } \mathrm{m} ; \\ \mathrm{D} & \text { kinetic mass transfer coefficient, } \mathrm{m} / \mathrm{s} ; \\ \omega_{k} & \text { diffusion coefficient, } \mathrm{m}^{2} / \mathrm{s} ;\end{array}$

